\title{
Integral representation of the scalar propagators on the de Sitter expanding universe
}

\author{
Ion I. Cotăescu ${ }^{\text {a }}$, Ion Cotăescu Jr. \\ West University of Timişoara, V. Pârvan Ave. 4, 300223 Timisoara, Romania
}

Received: 4 June 2019 / Accepted: 29 July 2019 / Published online: 9 August 2019

(C) The Author(s) 2019

\begin{abstract}
A new type of integral representation is proposed for the propagators of the massive Klein-Gordon field minimally coupled to gravity of the de Sitter expanding universe. This representation encapsulates the effects of the Heaviside step functions of the Feynman propagators, making possible for the first time the calculation of Feynman diagrams involving scalar particles on this background. In order to convince the reader, a simple example is given outlining as a premiere the amplitudes in the second order of perturbations of the Compton effect in the de Sitter scalar quantum electrodynamics.
\end{abstract}

\section{Introduction}

The classical or quantum scalar fields are the principal pieces used in various models of curved spacetime. Of a special interest in cosmology is the de Sitter expanding universe carrying scalar fields variously coupled to gravity whose quantum modes can be analytically solved [1-6]. Nevertheless, despite this opportunity, we have not yet a complete scalar quantum field theory (QFT) on de Sitter backgrounds based on perturbations and renormalization procedures able to describe all the processes involving scalar bosons. This is because of the technical difficulties in calculating Feynman diagrams affecting the fields of any spin on the de Sitter expanding universe.

The source of these difficulties is the fact that the causal propagators, expressed explicitly in terms of Heaviside step functions depending on time, lead to the fragmentation of the time integrals of the chronological products of free fields giving the transition amplitudes in different orders of perturbations. Under such circumstances, these integrals cannot be evaluated, forcing one to restrict oneself so far only to the first order amplitudes of the de Sitter QFT which do not involve propagators [7-17]. Note that the processes in the first order

a e-mail: icotaescu@yahoo.com of perturbations which are forbidden in special relativity by the energy-momentum conservation are allowed on the de Sitter spacetimes where the momentum and energy cannot be measured simultaneously [18]. However, the calculations in the first order of perturbations are only the first step to a complete QFT involving propagators for which we must get over the above-mentioned difficulties.

In the traditional QFT on the Minkowskian spacetime this problem is solved by the Fourier representations of the causal propagators, which encapsulate the effect of the Heaviside step functions [19]. Unfortunately, in the de Sitter case such Fourier representations do not hold as we shall explain in what follows. Therefore, we must look for another type of integral representation able to take over the effects of the Heaviside functions. Recently, we succeeded to find a new integral representation of the propagators of the Dirac field on the de Sitter [20] or any spatially flat FLRW [21] spacetimes which is different from the usual Fourier integrals allowed in special relativity. Here we would like to continue this study applying the same method to the massive and charged KleinGordon fields, minimally coupled to gravity of the de Sitter expanding universe, writing down for the first time the new integral representation of their propagators.

Moreover, we show that this new integral representation plays the same role as the familiar Fourier one in special relativity, helping us to calculate the Feynman diagrams of the de Sitter scalar quantum electrodynamics (SQED) in a similar manner to the flat case. In order to convince the reader we present as a premiere the amplitudes of the Compton effect on the de Sitter expanding universe, written in a closed form thanks to our integral representation.

We start in the second section presenting briefly the massive scalar field whose mode functions are written in the conformal local chart with Cartesian coordinates. The next section is devoted to our principal result reported here, demonstrating that the integral representation we propose gives just the Feynman propagator after applying the method of con- 
tour integrals [19]. In the third section we derive for the first time the amplitudes of the Compton effect on the de Sitter expanding portion. Finally some concluding remarks are presented.

\section{Massive scalar field}

Let us consider the de Sitter expanding universe defined as the expanding portion of the $(1+3)$-dimensional de Sitter manifold, equipped with the spatially flat FLRW chart whose coordinates, $x^{\mu}(\alpha, \ldots, \mu, \nu, \ldots=0,1,2,3)$, are the proper time $x^{0}=t$ and the Cartesian coordinates $x^{i}$ $(i, j, k, \ldots=1,2,3)$, for which we use the vector notation, $\mathbf{x}=\left(x^{1}, x^{2}, x^{3}\right)$. For technical reasons we work here mainly in the conformal chart having the conformal time

$t_{c}=-\frac{1}{\omega} \mathrm{e}^{-\omega t}<0$,

and the same space coordinates. In these charts the line element reads [22]

$$
\begin{aligned}
\mathrm{d} s^{2}=g_{\mu \nu}(x) \mathrm{d} x^{\mu} \mathrm{d} x^{\nu} & =\mathrm{d} t^{2}-a(t)^{2}(\mathrm{~d} \mathbf{x} \cdot \mathrm{d} \mathbf{x}) \\
& =a\left(t_{c}\right)^{2}\left(\mathrm{~d} t_{c}^{2}-\mathrm{d} \mathbf{x} \cdot \mathrm{d} \mathbf{x}\right),
\end{aligned}
$$

where

$a(t)=\mathrm{e}^{\omega t} \rightarrow a\left(t_{c}\right)=-\frac{1}{\omega t_{c}}$,

depending on the Hubble constant of the de Sitter spacetime denoted here by $\omega$.

In the conformal chart, the Klein-Gordon equation of a charged scalar field of mass $m$ takes the form

$\left(\partial_{t_{c}}^{2}-\Delta-\frac{2}{t_{c}} \partial_{t_{c}}+\frac{m^{2}}{\omega^{2} t_{c}^{2}}\right) \phi\left(t_{c}, \mathbf{x}\right)=0$,

allowing for the well-known solutions that can be expanded in terms of plane waves of positive and negative frequencies as [22]

$$
\begin{aligned}
\phi(x) & =\phi^{(+)}(x)+\phi^{(-)}(x) \\
& =\int \mathrm{d}^{3} p\left[f_{\mathbf{p}}(x) a(\mathbf{p})+f_{\mathbf{p}}^{*}(x) b^{*}(\mathbf{p})\right],
\end{aligned}
$$

where the fundamental solutions have the general form

$f_{\mathbf{p}}(x)=f_{\mathbf{p}}\left(t_{c}, \mathbf{x}\right)=\frac{1}{\sqrt{\pi \omega}} \frac{\mathrm{e}^{i \mathbf{p} \cdot \mathbf{x}}}{\left[2 \pi a\left(t_{c}\right)\right]^{\frac{3}{2}}} \mathscr{F}_{\nu}\left(t_{c}\right)$.

The time modulation function $\mathscr{F}_{v}$ may be any arbitrary linear combination of Bessel functions. In what follows it is convenient to consider modified Bessel functions $K_{v}$ instead of the usual Hankel ones, such that we can write

$\mathscr{F}_{v}\left(t_{c}\right)=c_{1} K_{i v}\left(i p t_{c}\right)+c_{2} K_{i v}\left(-i p t_{c}\right)$,

where $v=\sqrt{\frac{m^{2}}{\omega^{2}}-\frac{9}{4}}$ (in the minimal coupling) while $c_{1}$ and $c_{2}$ are arbitrary complex valued constants.

The fundamental solutions must satisfy the orthonormalization relations

$$
\begin{aligned}
\left\langle f_{\mathbf{p}}, f_{\mathbf{p}^{\prime}}\right\rangle=-\left\langle f_{\mathbf{p}}^{*}, f_{\mathbf{p}^{\prime}}^{*}\right\rangle & =\delta^{3}\left(\mathbf{p}-\mathbf{p}^{\prime}\right), \\
\left\langle f_{\mathbf{p}}, f_{\mathbf{p}^{\prime}}^{*}\right\rangle & =0,
\end{aligned}
$$

with respect to the relativistic scalar product [22]

$\left\langle\phi, \phi^{\prime}\right\rangle=i \int \frac{\mathrm{d}^{3} x}{\left(\omega t_{c}\right)^{2}} \phi^{*}(x) \stackrel{\leftrightarrow}{\partial_{t_{c}}} \phi^{\prime}(x)$

that hold only if we set

$\left|c_{1}\right|^{2}-\left|c_{2}\right|^{2}=1$,

as it results from Eqs. (10) and (1). These constants define the vacuum $[23,24]$ as in the case of the Bunch-Davies vacuum [25], where $c_{1}=1$ and $c_{2}=0$, which is a particular case of adiabatic vacuum [26-34]. In what follows we use this vacuum for brevity but all our formulas we derive here can be rewritten easily in any other vacuum.

After fixing he constants $c_{1}$ and $c_{2}$ the quantization can be done in a canonical manner replacing the wave functions of the field (5) by field operators, $a(\mathbf{p}) \rightarrow \mathfrak{a}(\mathbf{p})$ and $b(\mathbf{p}) \rightarrow \mathfrak{b}(\mathbf{p})$, such that $b^{*} \rightarrow \mathfrak{b}^{\dagger}[19]$. Then we assume that the particle $\left(\mathfrak{a}, \mathfrak{a}^{\dagger}\right)$ and antiparticle $\left(\mathfrak{b}, \mathfrak{b}^{\dagger}\right)$ operators fulfill the standard commutation relations in the momentum representation, among which the non-vanishing ones are

$\left[a(\mathbf{p}), a^{\dagger}\left(\mathbf{p}^{\prime}\right)\right]=\left[b(\mathbf{p}), b^{\dagger}\left(\mathbf{p}^{\prime}\right)\right]=\delta^{3}\left(\mathbf{p}-\mathbf{p}^{\prime}\right)$.

In the configuration representation the partial commutator functions of positive or negative frequencies,

$i D^{( \pm)}\left(x, x^{\prime}\right)=\left[\phi^{( \pm)}(x), \phi^{( \pm) \dagger}\left(x^{\prime}\right)\right]$

give the total one, $D=D^{(+)}+D^{(-)}$. These functions are solutions of the Klein-Gordon equation in both sets of variables and obey $\left[D^{( \pm)}\left(x, x^{\prime}\right)\right]^{*}=D^{(\mp)}\left(x, x^{\prime}\right)$ such that $D$ is a real valued function. These functions can be written as mode integrals as

$$
\begin{aligned}
i D^{(+)}\left(x, x^{\prime}\right) & =i D^{(+)}\left(t_{c}, t_{c}^{\prime}, \mathbf{x}-\mathbf{x}^{\prime}\right) \\
& =\int \mathrm{d}^{3} p f_{\mathbf{p}}(x) f_{\mathbf{p}}\left(x^{\prime}\right)^{*}
\end{aligned}
$$




$$
\begin{aligned}
= & \frac{1}{\pi \omega} \frac{1}{\left[4 \pi^{2} a\left(t_{c}\right) a\left(t_{c}^{\prime}\right)\right]^{\frac{3}{2}}} \\
& \times \int \mathrm{d}^{3} p e^{i \mathbf{p} \cdot\left(\mathbf{x}-\mathbf{x}^{\prime}\right)} K_{i v}\left(i p t_{c}\right) K_{i v}\left(-i p t_{c}^{\prime}\right),
\end{aligned}
$$

$$
\begin{aligned}
i D^{(-)}\left(x, x^{\prime}\right)= & i D^{(-)}\left(t_{c}, t_{c}^{\prime}, \mathbf{x}-\mathbf{x}^{\prime}\right) \\
= & -\int \mathrm{d}^{3} p f_{\mathbf{p}}(x)^{*} f_{\mathbf{p}}\left(x^{\prime}\right) \\
= & -\frac{1}{\pi \omega} \frac{1}{\left[4 \pi^{2} a\left(t_{c}\right) a\left(t_{c}^{\prime}\right)\right]^{\frac{3}{2}}} \\
& \times \int \mathrm{d}^{3} p \mathrm{e}^{i \mathbf{p} \cdot\left(\mathbf{x}-\mathbf{x}^{\prime}\right)} K_{i v}\left(-i p t_{c}\right) K_{i v}\left(i p t_{c}^{\prime}\right),
\end{aligned}
$$

taking similar forms after changing $\mathbf{p} \rightarrow-\mathbf{p}$ in the last integral. Note that these integrals can be solved in terms of hypergeometric functions obtaining well-known closed formulas [22].

\section{Integral representations of the scalar propagators}

The commutator functions allow us to construct the propagators, i.e., the Green functions corresponding to initial conditions at $t= \pm \infty$, without solving the Green equation. As in the scalar theory on Minkowski spacetime, we may use the Heaviside step functions for defining the retarded, $D_{\mathrm{R}}$, and advanced, $D_{\mathrm{A}}$, propagators,

$$
\begin{aligned}
& D_{\mathrm{R}}\left(t_{c}, t_{c}^{\prime}, \mathbf{x}-\mathbf{x}^{\prime}\right)=\theta\left(t_{c}-t_{c}^{\prime}\right) D\left(t_{c}, t_{c}^{\prime}, \mathbf{x}-\mathbf{x}^{\prime}\right), \\
& D_{\mathrm{A}}\left(t_{c}, t_{c}^{\prime}, \mathbf{x}-\mathbf{x}^{\prime}\right)=-\theta\left(t_{c}^{\prime}-t_{c}\right) D\left(t_{c}, t_{c}^{\prime}, \mathbf{x}-\mathbf{x}^{\prime}\right),
\end{aligned}
$$

while the causal Feynman propagator has the well-known form [19],

$$
\begin{aligned}
i D_{F}\left(t_{c}, t_{c}^{\prime}, \mathbf{x}-\mathbf{x}^{\prime}\right) & =\left\langle 0\left|T\left[\phi(x) \phi^{\dagger}\left(x^{\prime}\right)\right]\right| 0\right\rangle \\
& =\theta\left(t_{c}-t_{c}^{\prime}\right) D^{(+)}\left(t_{c}, t_{c}^{\prime}, \mathbf{x}-\mathbf{x}^{\prime}\right) \\
& -\theta\left(t_{c}^{\prime}-t_{c}\right) D^{(-)}\left(t_{c}, t_{c}^{\prime}, \mathbf{x}-\mathbf{x}^{\prime}\right) .
\end{aligned}
$$

Our main goal here is to find the suitable integral representation of these propagators which should encapsulate the effect of the Heaviside step functions.

As mentioned, the propagators (16), (17) and (18) cannot be used in the concrete calculations of Feynman diagrams as they stay because of their explicit dependence on the Heaviside $\theta$-functions. In the case of the Minkowski spacetime this problem is solved by representing these propagators as 4-dimensional Fourier integrals, which take over the effects of the Heaviside functions according to the method of the contour integrals [19]. In this manner one obtains a suitable integral representation of the Feynman propagators allowing one to work exclusively in the momentum representation.
In de Sitter spacetimes we also have a momentum representation, but we do not know how to exploit it since in this geometry the propagators are functions of two time variables, $t-t^{\prime}$ and $t t^{\prime}$, instead of the unique variable $t-t^{\prime}$ of the Minkowski case. This situation generates new difficulties, since apart from a Fourier transform in $t-t^{\prime} \in \mathbb{R}$ a supplementary Mellin transform for the new variable $t t^{\prime} \in \mathbb{R}^{+}$ [35] might be considered. Obviously, an integral with two more variables of integration is not a convenient solution for representing the Feynman propagators.

This means that we must look for an alternative integral representation based on the method of the contour integrals [19] but avoiding the mentioned Fourier or Mellin transforms. As in the Dirac case [20,21], the explicit forms of the partial commutator functions (14) and (15) suggest us to postulate the following integral representation of the Feynman propagator:

$$
\begin{aligned}
D_{F}\left(x, x^{\prime}\right) \equiv & D_{F}\left(t_{c}, t_{c}^{\prime}, \mathbf{x}-\mathbf{x}^{\prime}\right)=\frac{1}{\pi^{2} \omega} \frac{1}{\left[4 \pi^{2} a\left(t_{c}\right) a\left(t_{c}^{\prime}\right)\right]^{\frac{3}{2}}} \\
& \times \int \mathrm{d}^{3} p \mathrm{e}^{i \mathbf{p} \cdot\left(\mathbf{x}-\mathbf{x}^{\prime}\right)} \int_{-\infty}^{\infty} d s \\
& |s| \frac{K_{i v}\left(i s t_{c}\right) K_{i v}\left(-i s t_{c}^{\prime}\right)}{s^{2}-p^{2}-i \epsilon}
\end{aligned}
$$

It remains to prove that this integral representation gives just the Feynman propagator (18) according to the well-known method of contour integrals [19]. Focusing on the last integral of Eq. (19) denoted as

$\mathscr{I}\left(t_{c}, t_{c}^{\prime}\right)=\int_{-\infty}^{\infty} \mathrm{d} s M\left(s, t_{c}, t_{c}^{\prime}\right)$

we observe that for large values of $|s|$ the modified Bessel functions can be approximated as in Eq. (2), obtaining the asymptotic behavior

$M\left(s, t_{c}, t_{c}^{\prime}\right) \sim \frac{\mathrm{e}^{-i s\left(t_{c}-t_{c}^{\prime}\right)}}{s}$.

Now we can estimate the integrals on the semicircular parts, $c_{ \pm}$, of the contours pictured in Fig. 1 taking $s \sim R e^{i \varphi}$ and using Eq. (3.338-6) of Ref. [35], which gives

$\int_{c_{ \pm}} \mathrm{d} s M\left(s, t_{c}, t_{c}^{\prime}\right) \sim I_{0}\left[ \pm R\left(t_{c}-t_{c}^{\prime}\right)\right] \sim \frac{1}{\sqrt{R}} \mathrm{e}^{ \pm R\left(t_{c}-t_{c}^{\prime}\right)}$

since the modified Bessel function $I_{0}$ behaves as in the first of Eq. (2). In the limit of $R \rightarrow \infty$ the contribution of the semicircle $c_{+}$vanishes for $t_{c}^{\prime}>t_{c}$, while the one of the semicircle $c_{-}$vanishes for $t_{c}>t_{c}^{\prime}$. Therefore, the integration along the real $s$-axis is equivalent to the following contour integrals: 


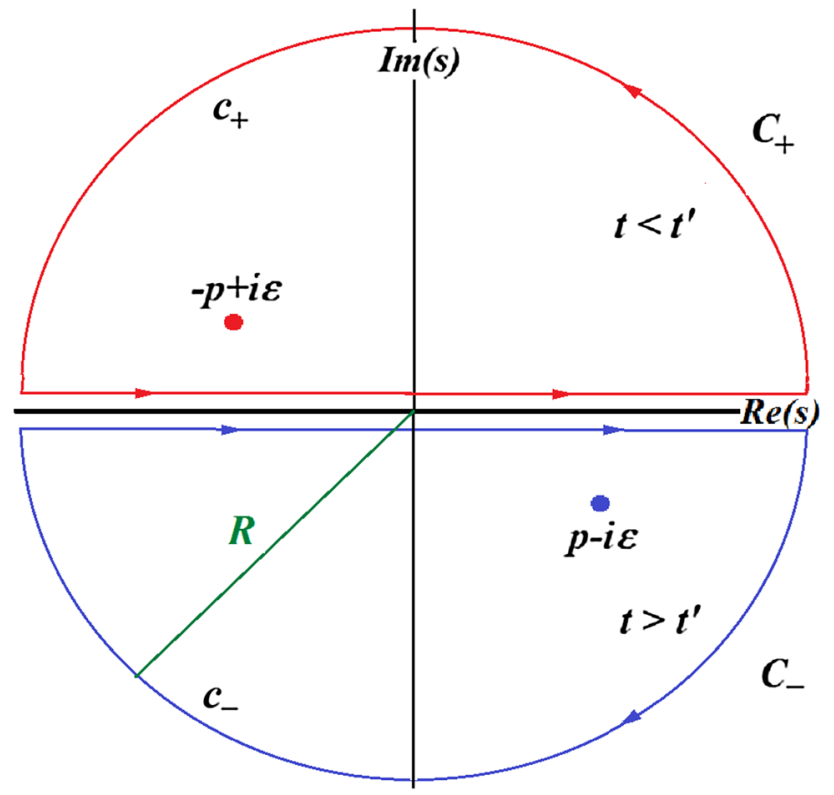

Fig. 1 The contours of integration in the complex $s$-plane, $C_{ \pm}$, are the limits of the pictured ones for $R \rightarrow \infty$

$\mathscr{I}\left(t_{c}, t_{c}^{\prime}\right)=\left\{\begin{array}{l}\int_{C_{+}} \mathrm{d} s M\left(s, t_{c}, t_{c}^{\prime}\right)=\mathscr{I}_{+}\left(t_{c}, t_{c}^{\prime}\right) \text { for } t_{c}<t_{c}^{\prime}, \\ \int_{C_{-}} \mathrm{d} s M\left(s, t_{c}, t_{c}^{\prime}\right)=\mathscr{I}_{-}\left(t_{c}, t_{c}^{\prime}\right) \text { for } t_{c}>t_{c}^{\prime},\end{array}\right.$

where the contours $C_{ \pm}$are the limits for $R \rightarrow \infty$ of those of Fig. 1. Then we may apply Cauchy's theorem [36],

$\mathscr{I}_{ \pm}\left(t_{c}, t_{c}^{\prime}\right)= \pm\left. 2 \pi i \operatorname{Res}\left[M\left(s, t_{c}, t_{c}^{\prime}\right)\right]\right|_{s=\mp p \pm i \epsilon}$,

taking into account that in the simple poles at $s= \pm p \mp i \epsilon$ we have the residues

$\left.\operatorname{Res}\left[M\left(s, t, t^{\prime}\right)\right]\right|_{s= \pm p \mp i \epsilon}= \pm \frac{1}{2} K_{v}\left( \pm i p t_{c}\right) K_{v}\left(\mp i p t_{c}^{\prime}\right)$.

Consequently, the integral $\mathscr{I}_{-}\left(t_{c}, t_{c}^{\prime}\right)$ gives the first term of the Feynman propagator (18) with $D^{(+)}$expanded as in Eq. (14), while the integral $\mathscr{I}_{+}\left(t_{c}, t_{c}^{\prime}\right)$ yields its second term with $D^{(-)}$in the form (15), proving that the integral representation (19) is correct.

The other propagators, $D_{\mathrm{A}}$ and $D_{\mathrm{R}}$, can be represented in a similar manner by changing the positions of the poles as in the flat case [19],

$$
\begin{aligned}
\underset{\mathrm{A}}{D_{\mathrm{R}}\left(x, x^{\prime}\right)} & =\underset{\mathrm{A}}{D_{\mathrm{R}}\left(t_{c}, t_{c}^{\prime}, \mathbf{x}-\mathbf{x}^{\prime}\right)} \\
& =\frac{1}{\pi^{2} \omega} \frac{1}{\left[4 \pi^{2} a\left(t_{c}\right) a\left(t_{c}^{\prime}\right)\right]^{\frac{3}{2}}}
\end{aligned}
$$

$$
\begin{aligned}
& \times \int d^{3} p e^{i \mathbf{p} \cdot\left(\mathbf{x}-\mathbf{x}^{\prime}\right)} \\
& \int_{-\infty}^{\infty} \mathrm{d} s|s| \frac{K_{i v}\left(i s t_{c}\right) K_{i v}\left(-i s t_{c}^{\prime}\right)}{(s \pm i \epsilon)^{2}-p^{2}},
\end{aligned}
$$

but in our integral representation instead of the Fourier one.

Finally, we note that the above integral representations can be rewritten at any time in the FLRW chart, $\{t, \mathbf{x})\}$, substituting $t_{c} \rightarrow t$ and $a\left(t_{c}\right) \rightarrow a(t)$ according to Eqs. (1) and (3).

\section{Example: Compton effect in SQED}

We succeeded thus in deriving the specific integral representations of the scalar propagators on the de Sitter expanding universe that can be used for calculating the Feynman diagrams of the physical effects involving the Klein-Gordon field. Here we give a simple example outlining how our approach works in the SQED on the de Sitter expanding universe, deriving the amplitudes of the Compton effect in the second order of perturbations.

We consider our massive charged scalar field $\phi$ to be coupled minimally to the electromagnetic field $A_{\mu}$ through the interaction Lagrangian

$\mathscr{L}_{\text {int }}=-i e \sqrt{g(x)} g^{\mu \nu}(x) A_{\mu}(x)\left[\phi^{\dagger}(x) \stackrel{\leftrightarrow}{\partial_{\nu}} \phi(x)\right]$,

where $e$ is the electrical charge. We know that in the chart $\left\{t_{c}, \mathbf{x}\right\}$ the electromagnetic potential can be expanded in terms of plane waves in a similar manner to the Minkowski spacetime since the Maxwell equations are conformally invariant. However, the Lorentz condition does not have this property in any gauge such that we are forced to work exclusively in the Coulomb gauge where the condition $A_{0}=0$ complies with the conformal symmetry $[15,37]$. Therefore, we may write the expansion

$$
A_{i}(x)=\int \mathrm{d}^{3} k \sum_{\lambda}\left[\mu_{\mathbf{k}, \lambda ; i}(x) \alpha(\mathbf{k}, \lambda)+\mu_{\mathbf{k}, \lambda ; i}(x)^{*} \alpha^{\dagger}(\mathbf{k}, \lambda)\right],
$$

in terms of the mode functions,

$\mu_{\mathbf{k}, \lambda ; i}\left(t_{c}, \mathbf{x}\right)=\frac{1}{(2 \pi)^{3 / 2}} \frac{1}{\sqrt{2 k}} \mathrm{e}^{-i k t_{c}+i \mathbf{k} \cdot \mathbf{x}} \varepsilon_{i}(\mathbf{k}, \lambda)$,

depending on the components of the polarization vectors $\varepsilon_{i}(\mathbf{k}, \lambda)$ of momentum $\mathbf{k}(k=|\mathbf{k}|)$ and helicity $\lambda= \pm 1$ [15]. Note that in this gauge the unphysical components are eliminated leaving us only with a space polarization vector, which is orthogonal to the momentum one, $k^{i} \varepsilon_{i}(\mathbf{k}, \lambda)=0$. 
Then the quantization of the free Maxwell field can be done postulating the commutation rules

$\left[\alpha(\mathbf{k}, \lambda), \alpha^{\dagger}\left(\mathbf{k}^{\prime}, \lambda^{\prime}\right)\right]=\delta_{\lambda, \lambda^{\prime}} \delta^{3}\left(\mathbf{k}-\mathbf{k}^{\prime}\right)$

which have the same form as in the flat case.

Furthermore, the theory of interacting fields can be constructed by using the reduction formalism and the canonical quantization in Coulomb gauge as in the case of our QED [15]. In this manner we obtain a coherent theory in which the in-out transitions are performed by unitary operators.

With these preparations we can write the first of the pair of the Compton amplitudes [19] in a self-explanatory notation as

$$
\begin{aligned}
\mathscr{A}_{\lambda_{1}, \lambda_{2}} & \left(\mathbf{p}_{1}, \mathbf{k}_{1}, \mathbf{p}_{2}, \mathbf{k}_{2}\right) \equiv\left\langle\text { out } \mathbf{p}_{2},\left(\mathbf{k}_{2}, \lambda_{2}\right)\right| \text { in } \mathbf{p}_{1},\left(\mathbf{k}_{1}, \lambda_{1}\right\rangle \\
= & -i \frac{e^{2}}{2} \int d^{4} x d^{4} x^{\prime} \sqrt{g(x) g\left(x^{\prime}\right)} g^{i j}(x) g^{k l}\left(x^{\prime}\right) \\
& \times \mu_{\mathbf{k}_{2}, \lambda_{2}, j}(x)^{*} \mu_{\mathbf{k}_{1}, \lambda_{1}, l}\left(x^{\prime}\right) \\
& {\left[f_{\mathbf{p}_{2}}^{*}(x) \stackrel{\leftrightarrow}{\partial_{i}} D_{F}\left(x, x^{\prime}\right) \overleftrightarrow{\partial_{k}^{\prime}} f_{\mathbf{p}_{1}}\left(x^{\prime}\right)\right], }
\end{aligned}
$$

where $D_{F}$ is given by our integral representation (19). We perform first the space integrals generating Dirac $\delta$-functions, which have to ensure the momentum conservation after integrating over the internal momentum $\mathbf{p}$ of $D_{F}$. Thus, after a little calculation we may write

$$
\begin{aligned}
& \mathscr{A}_{\lambda_{1}, \lambda_{2}}\left(\mathbf{p}_{1}, \mathbf{k}_{1}, \mathbf{p}_{2}, \mathbf{k}_{2}\right)=-i \frac{e^{2}}{2} \delta^{3}\left(\mathbf{p}_{1}+\mathbf{k}_{1}-\mathbf{p}_{2}-\mathbf{k}_{2}\right) \\
& \quad \times p_{1}^{i} \varepsilon_{i}\left(\mathbf{k}_{1}, \lambda_{1}\right) p_{2}^{j} \varepsilon_{j}\left(\mathbf{k}_{2}, \lambda_{2}\right)^{*} \\
& \quad \times \int_{-\infty}^{\infty} \mathrm{d} s|s| \frac{\mathscr{V}\left(p_{2}, k_{2}, s\right)^{*} \mathscr{V}\left(p_{1}, k_{1}, s\right)}{s^{2}-\left|\mathbf{p}_{1}+\mathbf{k}_{1}\right|^{2}-i \epsilon}
\end{aligned}
$$

where we introduced the vertex functions defined up to a phase factor:

$$
\begin{aligned}
\mathscr{V}(p, k, s)= & \frac{1}{2 \omega^{2} \pi^{3} \sqrt{k}} \\
& \times \int_{0}^{\infty} \mathrm{d} \tau K_{i v}\left(-i \frac{p}{\omega} \tau\right) K_{i v}\left(i \frac{s}{\omega} \tau\right) \mathrm{e}^{i \frac{k}{\omega} \tau},
\end{aligned}
$$

after changing the variable of integration $t_{c} \rightarrow \tau=-\omega t_{c}$. We obtained thus a closed form of the first Compton amplitude, bearing in mind that the second one can be obtained directly by changing $\mathbf{k}_{1} \leftrightarrow \mathbf{k}_{2}$ in Eq. (31) [19]. We must stress that this result is due to our integral representation, since otherwise the Heaviside step functions of the original form (18) would have mixed up the time integrals.

The Compton amplitudes obtained here are complicated since, in general, the quantum effects in de Sitter spacetimes are described by formulas involving integrals of the form (32). For example, in the de Sitter QED in Coulomb gauge
[15] the amplitudes in the first order of perturbations are given by integrals of this form whose analysis would require an extended analytical and numerical study [15-17]. A similar study can be performed in the case of the Compton effect calculated here but this exceeds the purposes of this paper where the principal objective was to define our new integral representation of the scalar propagators.

\section{Concluding remarks}

The above example shows that the integral representation of the scalar propagators proposed here is crucial for calculating the Feynman diagrams in any order of the SQED in the presence of the gravity of the de Sitter background. Thus one could find new observable effects involving interacting fields, allowed by the local gravity, whose indirect influence could be better measured than its direct interaction with the quantum matter, which is very weak.

It remains to study the renormalization, observing that here it is not certain that the standard regularization procedures, as for example the Pauli-Villars method, will work as in the flat case. This is because of the structure of the propagators studied here which depend on the mass only indirectly through the index of the $K$-functions. Thus a priority task is to find suitable methods of regularization and renormalization looking for alternative methods or adapting the well-known regularization procedures of the two-point functions [38-42].

Concluding we can say with moderate optimism that now we have all the tools we need for calculating at least the nongravitational effects of the massive scalar field in the presence of gravity of the de Sitter expanding universe.

Data Availability Statement This manuscript has no associated data or the data will not be deposited. [Authors' comment: There are no experimental data. All the other data are public and can be deposited, of course.]

Open Access This article is distributed under the terms of the Creative Commons Attribution 4.0 International License (http://creativecomm ons.org/licenses/by/4.0/), which permits unrestricted use, distribution, and reproduction in any medium, provided you give appropriate credit to the original author(s) and the source, provide a link to the Creative Commons license, and indicate if changes were made.

Funded by SCOAP . $^{3}$

\section{Appendix A: Modified Bessel functions}

The modified Bessel functions $I_{v}(z)$ and $K_{v}(z)=K_{-v}(z)$ are related to the Hankel ones such that their Wronskian [43] gives the identity

$$
K_{v}(i s) \overleftrightarrow{\partial_{s}} K_{v}(-i s)=W\left[K_{v}(i s), K_{v}(-i s)\right]=\frac{i \pi}{s}
$$


For $|z| \rightarrow \infty$ and any $v$ we have

$I_{v}(z) \rightarrow \sqrt{\frac{\pi}{2 z}} e^{z}, \quad K_{v}(z) \rightarrow K_{\frac{1}{2}}(z)=\sqrt{\frac{\pi}{2 z}} e^{-z}$.

\section{References}

1. O. Nachtmann, Commun. Math. Phys. 6, 1 (1967)

2. N.A. Chernikov, E.A. Tagirov, Ann. Inst. Henri Poincaré (A) Physique théorique 9, 109 (1968)

3. G. Börner G., H.P.Dürr, Il Nuovo Cimento A, Ser. 10(64), 669 (1969)

4. E.A. Tagirov, Ann. Phys. 76, 561 (1973)

5. I.I. Cotăescu, C. Crucean, A. Pop, Int. J. Mod. Phys. A 23, 2563 (2008)

6. I.I. Cotăescu, G. Pascu, F.A. Dragoesc, Mod. Phys. A 28, 1350160 (2013)

7. K.-H. Lotze, Class. Quantum Gravity 4, 1437 (1987)

8. K.-H. Lotze, Class. Quantum Gravity 5, 595 (1988)

9. K.-H. Lotze, Nucl. Phys. B 312, 673 (1989)

10. I.L. Buchbinder, E.S. Fradkin, D.M. Gitman, Forstchr. Phys. 29, 187 (1981)

11. I.L. Buchbinder, L.I. Tsaregorodtsev, Int. J. Mod. Phys A 7, 2055 (1992)

12. L.I. Tsaregorodtsev, Russ. Phys. J. 41, 1028 (1989)

13. J. Audretsch, P. Spangehl, Class. Quantum Gravity 2, 733 (1985)

14. J. Audretsch, P. Spangehl, Phys. Rev. D 33, 997 (1986)

15. I.I. Cotăescu, C. Crucean, Phys. Rev. D 87, 044016 (2013)

16. C. Crucean, M.-A. Baloi, Phys. Rev. D 93, 044070 (2016)

17. C. Crucean, M.-A. Baloi, Int. J. Mod. Phys. A 32, 1750208 (2017)

18. I.I. Cotăescu, GRG 43, 1639 (2011)

19. S. Drell, J.D. Bjorken, Relativistic Quantum Fields (Mc Graw-Hill Book Co., New York, 1965)

20. I.I. Cotăescu, Eur. Phys. J. C 78, 769 (2018)
21. I.I. Cotăescu, Int. J. Mod. Phys. A 34, 1950024 (2019)

22. N.D. Birrel, P.C.W. Davies, Quantum Fields in Curved Space (Cambridge University Press, Cambridge, 1982)

23. L. Parker, Phys. Rev. Lett. 21, 562 (1968)

24. L. Parker, Phys. Rev. 183, 1057 (1969)

25. T.S. Bunch, P.C.W. Davies, Proc. R. Soc. Lond. 360, 117 (1978)

26. A.A. Grib, S.G. Mamaev, Yad. Fiz. 10, 1276 (1969)

27. A.A. Grib, S.G. Mamaev, Sov. J. Nucl. Phys. 10, 722 (1970)

28. Y.B. Zeldovich, Pisma Zh. Eksp. Teor. Fiz. 12, 443 (1970)

29. Y.B. Zeldovich, A.A. Starobinsky, Sov. Phys. JETP 34, 1159(1972)

30. Y.B. Zeldovich, A.A. Starobinsky, Zh Eksp, Teor. Fiz. 61, 2161 (1971)

31. A.A. Grib, S.G. Mamaev, V.M. Mostepanenko, Gen. Relat. Gravity 7, 535 (1976)

32. A.A. Grib, S.G. Mamaev, V.M. Mostepanenko, J. Phys. A Gen. Phys. 13, 2057 (1980)

33. B. Allen, Phys. Rev. D 32(12), 3136-3149 (1985)

34. R. Bousso, A. Maloney, A. Strominger, Phys. Rev. D 65, 104039 (2002). arXiv:hep-th/0112218

35. I.S. Gradshteyn, I.M. Ryzhik, Table of Integrals, Series, and Products (Academic Press, New York, 2007)

36. L.V. Ahlfors, Complex analysis: an introduction to the theory of analytic functions of one complex variable (McGraw-Hill, New York, London, 1953)

37. I.I. Cotăescu, C. Crucean, Prog. Theor. Phys. 124, 1051 (2010)

38. J.S. Schwinger, Phys. Rev. D 82, 664 (1951)

39. J.S. Schwinger, J. Math. Phys. 2, 407 (1961)

40. L.V. Keldysh, Sov. Phys. JETP 20, 1018 (1965)

41. B.S. DeWitt, Phys. Rep. C 19, 295 (1975)

42. L.S. Brown, Phys. Rev. D 15, 1469 (1977)

43. F.W.J. Olver, D.W. Lozier, R.F. Boisvert, C.W. Clark, NIST Handbook of Mathematical Functions (Cambridge University Press, Cambridge, 2010) 